I BIENAL LATINOAMERICANA DIDH

\title{
La sustancialidad de la Convención Belém do Pará para la superación de la discriminación estructural y la violencia contra la mujer fundada en el género
}

\author{
The substantiality of the Belém do Pará Convention to overcome structural \\ discrimination and gender-based violence against women
}

\section{Claudia Iriarte Rivas (iD \\ Universidad de Chile}

\begin{abstract}
RESUMEN En este trabajo se revisa el aporte y avance que ha significado la Convención Belém do Pará como instrumento del derecho internacional de los derechos humanos en el avance al respeto de estos derechos respecto de la mujer y en el logro de la superación de la violación de los derechos de la mujer. A partir de ello, se quiere destacar el aporte que este instrumento ha tenido en la conformación de un corpus normativo y en el desarrollo de estándares en materia de derechos humanos de la mujer, los cuales se han constituido como un cuerpo jurídico común en el Sistema Interamericano de Derecho Humanos, que puede y debe ser recepcionado por Estados que integran el sistema regional de derechos humanos.
\end{abstract}

PALABRAS CLAVE Convención Belém do Pará, derechos humanos de la mujer, violencia contra la mujer, discriminación estructural.

ABSTRACT This paper analyzes the contribution and progress produced by the Belém do Pará Convention as an instrument of international human rights law in terms of fostering the respect of these rights for women and in overcoming the violation of women's rights. Based on this, will highlight the contribution that this instrument has made to the creation of a regulatory framework and the development of standards in the area of women's human rights, which have become a common legal body in the Inter-American Human Rights System, which can and should be accepted by the States belonging to the regional human rights system.

KEYWORDS Belém do Pará Convention, women’s human rights, violence against women, structural discrimination. 


\section{Introducción}

La Convención Interamericana para Prevenir, Sancionar y Erradicar la Violencia Contra la Mujer "Convención de Belém do Pará» ${ }^{1}$ fue el primer instrumento internacional de carácter convencional que abordó los derechos humanos de las mujeres y la violencia de género.

En este trabajo revisaremos el aporte y avance que ha significado la Convención como instrumento del derecho internacional de los derechos humanos, para asegurar el respeto irrestricto de estos derechos respecto de la mujer, para el logro de la superación de la violación de sus derechos y la prevención, sanción y erradicación de la violencia contra la mujer. A partir de ello, se destacaremos el aporte de este texto en la conformación de un corpus normativo y en el desarrollo de estándares en materia de derechos humanos de las mujeres, que constituyen un cuerpo jurídico común en el Sistema Interamericano de Derecho Humanos (SIDH), que puede y debe ser recepcionado por Estados que integran el sistema regional de derechos humanos.

Revisaremos este instrumento desde tres perspectivas:

En primer lugar, revisaremos qué significó la adopción por parte del SIDH, en el contexto del derecho internacional de derechos humanos, del primer instrumento de carácter convencional que abordara de un modo específico la violencia contra la mujer por razones de género y la afectación que esta realidad implica al reconocimiento, goce y ejercicio de los derechos humanos y libertades fundamentales.

En lo referente a su contenido, se abordarán sus principales y más destacables aportes concernientes a la prevención, sanción y erradicación de la violencia contra la mujer. Se analizan aquí cuatro aspectos: la conceptualización de la violencia contra la mujer como violencia estructural, la vinculación de la violencia contra la mujer y los derechos humanos, y la recepción de la doctrina de la «diligencia debida» por parte de los Estados y los mecanismos de control que contempla.

Por último, se examinará de modo específico las disposiciones contenidas en este instrumento referidas a derechos protegidos y, de modo particular, respecto de la conceptualización y alcance del planteamiento que contiene la Convención Belém do Pará que señala que «toda mujer tiene derecho a un mundo libre de violencia». Su análisis se efectuará respecto de las disposiciones convencionales relativas a la conceptualización de violencia y al derecho de la mujer al reconocimiento, goce y ejercicios de los derechos humanos. Ello, de modo de sostener una interpretación posible, que plantee que la discriminación estructural contra la mujer es constitutiva de violencia contra la mujer en los términos que contempla la propia Convención.

1. Convención Interamericana para prevenir, sancionar y erradicar la violencia contra la Mujer, Organización de los Estados Americanos, 14 de agosto de 1995, disponible en https://bit.ly/2Qgy6QN. 


\section{La problemática de la violencia contra la mujer en el Sistema Internacional de los Derechos Humanos}

Hasta la adopción y ratificación de la Convención Belém do Pará, los únicos antecedentes en materia de violencia contra la mujer en el derecho internacional de los derechos humanos eran, primero, las Recomendaciones Generales 12 y 19, adoptadas en $1989^{2}$ y $1992^{3}$ por el Comité para la Eliminación de la Discriminación contra la Mujer (Comité CEDAW), ${ }^{4}$ ambas referidas a violencia contra la mujer. En segundo lugar, se encontraba la Declaración sobre la Eliminación de la Violencia contra la Mujer (DEVAW), acordada por la asamblea general en la $85 .{ }^{a}$ sesión plenaria del 20 de diciembre de $1993 .{ }^{5}$

La Convención contra la Eliminación de Todas las Formas de Discriminación Contra la Mujer (CEDAW) ${ }^{6}$ instrumento considerado como carta magna de los derechos de las mujeres (Jiménez Sánchez, 2018: 492), no aborda de modo especifico la violencia contra la mujer. Por ello, el Comité CEDAW, en virtud de su potestad para hacer sugerencias y recomendaciones de carácter general (artículo 21), adoptó recomendaciones generales sobre la violencia contra la mujer para suplir esta ausencia. La Recomendación General 12 se limita a recomendar que los Estados partes incluyan en sus informes periódicos información relativa a legislación y medidas adoptadas por ellos para erradicar la violencia contra la mujer. La Recomendación General 19, en cambio, plantea el abordaje de la violencia contra la mujer de un modo más sustancial, al considerarla como problemática central en materia de derechos humanos de la mujer. Así, en su numeral 4 señala: «Los informes de los Estados partes no siempre reflejaban [...] la estrecha vinculación entre la discriminación contra la mujer, la violencia contra la mujer, y las violaciones de los derechos humanos y las libertades

2. «Recomendación General 12: Violencia contra la mujer», Comité para la Eliminación de la Discriminación contra la Mujer, 1989, disponible en https://bit.ly/32grB6a.

3. «Recomendación General 19: La violencia contra la mujer», Comité para la Eliminación de la Discriminación contra la Mujer, 1992, disponible en https://bit.ly/3j8xGsp.

4. Los países que se han adherido al tratado tienen la obligación de presentar al Comité informes periódicos relativos a la aplicación de los derechos amparados por la Convención. El Comité formula recomendaciones generales y sugerencias, las que son remitidas a los Estados y tratan de artículos o temas que figuran en la Convención. También formula recomendaciones sobre cualquier cuestión que afecte a las mujeres que considere que los Estados partes deben dedicar más atención. Hasta la fecha, el Comité había aprobado 37 recomendaciones generales, publicadas en «Recomendaciones generales», Comité para la Eliminación de la Discriminación contra la Mujer, disponible en https://bit.ly/3j16awM.

5. «Declaración sobre la eliminación de todas las formas de violencia contra la mujer», Naciones Uni-

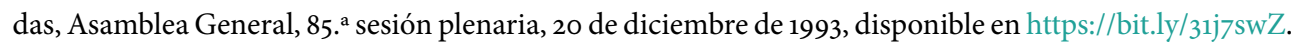

6. «Convención sobre la Eliminación de Todas las Formas de Discriminación Contra la Mujer», Resolución 34/180, Naciones Unidas, Asamblea General, 18 de diciembre de 1979, disponible en https:// bit.ly/2Qdocj9. 
fundamentales». Luego, en el numeral 7, agrega: «La violencia contra la mujer, que menoscaba o anula el goce de sus derechos humanos y sus libertades fundamentales en virtud del derecho internacional o de los diversos convenios de derechos humanos, constituye discriminación, como la define el artículo 1 de la Convención». Es decir, este instrumento internacional señala que la violencia contra la mujer está vinculada con el menoscabo, violación o anulación en el goce de sus derechos, y que su ocurrencia será considerada discriminación contra la mujer, según los criterios y definiciones establecidos en la propia CEDAW.

En julio de 2017, a 25 años de la adopción de la Resolución 19, fue aprobada una nueva Recomendación General 35, sobre la violencia por género contra la mujer, que actualiza la Recomendación 19. Conforme se establece en la propia introducción de esta recomendación, a 25 años de la Recomendación 19, se ofrece a los Estados una orientación adicional para «acelerar la eliminación de la violencia por razón de género contra la mujer», lo que es necesario, ya que, a pesar de los importantes avances en este periodo, esta violencia continúa siendo generalizada y tiene un alto grado de impunidad. La Recomendación 35 está concebida y estructurada como un complemento y profundización de la Recomendación 19, avanzando de un modo sustancial en la conceptualización y determinación de la violencia de género como un problema social que demanda respuestas integrales y cuya ocurrencia es uno de los medios sociales, políticos y económicos fundamentales a través de los cuales se perpetúa la posición subordinada de la mujer. ${ }^{8}$

La DEVAW, segundo instrumento internacional referido a derechos humanos y violencia contra la mujer, siguió muy de cerca a la Recomendación 19 en cuanto a la referencia contenida en el preámbulo respecto del carácter estructural de la violencia contra la mujer. Sin embargo, no profundizó en su desarrollo conceptual, ni avanzó en una propuesta que afrontara la prevención y sanción de la violencia contra la mujer desde una perspectiva de derechos humanos que aborde la discriminación. Se debe destacar que este texto, junto con las recomendaciones generales a la CEDAW, fue parte de un esfuerzo del Sistema Internacional de los Derechos Humanos en la década de los noventa de avanzar hacia la elaboración de instrumentos convencionales que abordaran la situación de violencia contra las mujeres basadas en su género.

7. «Recomendación General 35: La violencia por razón de género contra la mujer», Comité para la Eliminación de la Discriminación contra la Mujer, 2017, disponible en https://bit.ly/2EleB74.

8. De las medidas específicas que aborda, es posible destacar lo referido al establecimiento de las «Obligaciones de los Estados partes en relación con la violencia por razón de género contra la mujer». Las recomendaciones comprenden medidas legislativas generales, medidas preventivas, medidas de protección, medidas de enjuiciamiento y castigo, medidas de reparación, medidas de coordinación, vigilancia y recopilación de datos y medidas de cooperación internacional. Véase «Recomendación General 35». 
La Convención de Belém do Pará, como vemos, es el primer instrumento internacional de carácter convencional que aborda los derechos humanos de las mujeres y la violencia de género. Fue adoptada en junio de 1994 y entró en vigor internacional el 5 de marzo de $1995 .{ }^{9}$ Con ello, el Sistema Interamericano de Derechos Humanos crea un texto jurídico vinculante que aborda específicamente la violencia que se ejerce contra las mujeres, para llenar el vació existente en el Sistema Internacional de los Derechos Humanos.

En particular, aborda esta temática vinculándola con la afectación al reconocimiento, goce y ejercicio de los derechos humanos y libertades fundamentales (preámbulo y artículo 4); conceptualiza la violencia contra la mujer fundada en el género como violencia estructural e, incluso, plantea nuevas perspectivas y conceptualizaciones en materia de derechos humanos de la mujer, al reconocer el derecho de toda mujer a una vida libre de violencia y establecer la prohibición expresa al ejercicio de violencia basada en el género.

En el sistema europeo, el año 2011, el Consejo de Europa aprobó la Convención sobre Prevención y Lucha Contra la Violencia Contra las Mujeres y la Violencia Doméstica (Convenio de Estambul), la cual entró en vigor el año 2014. ${ }^{10}$ Sin pretender entrar a un análisis pormenorizado de este instrumento internacional, ya que en este trabajo nos abocamos al instrumento interamericano, es importante reseñar al menos sus características centrales y plantearse una mirada comparativa general del alcance de ambos instrumentos. El Convenio de Estambul, de modo similar que la Convención interamericana, tiene como antecedente las recomendaciones generales que complementan la CEDAW, e incluso la DEVAW, en el sentido de contar con una normativa específica que, desde la perspectiva de los derechos humanos, tenga por objetivo «la prevención y lucha contra la violencia contra las mujeres». Este es un texto extenso que, junto con el objetivo de proteger, prevenir, perseguir y eliminar la violencia contra las mujeres, plantea un marco global que guíe políticas y medidas de protección a las víctimas y, además, busca la promoción de la cooperación internacional para abordar y superar la violencia contra la mujer. El Convenio conceptualiza la violencia contra la mujer en términos amplios, definiéndola a partir de actos de violencia que se basan en el género y que puedan significar daño y sufrimiento a la mujer de variada índole, incluyendo el daño económico. Este último punto es en especial destacable, ya que ningún instrumento del derecho internacional de los derechos humanos referido a violencia señala de forma expresa esta forma de violencia.

9. Hasta la fecha, 32 Estados han ratificado el instrumento y 2 Estados miembros se han quedado al margen.

10. Hasta la fecha, 34 Estados han ratificado el instrumento, 11 solo han depositado el instrumento de firma y 2 se han quedado al margen. 
El punto más controvertido del Convenio de Estambul es que plantea la prevención y lucha contra la violencia contra las mujeres y la violencia doméstica como si fueran dos fenómenos de tal particularidad que requirieran ser abordados por separado, o bien que la violencia doméstica fuera una categoría diferente a la violencia de género (Ventura Franch, 2016: 203). Esta concepción se ve agravada al definir la violencia doméstica de un modo neutral, es decir, que cualquier integrante del núcleo doméstico puede ser víctima de violencia. Con ello, en definitiva la colocan como una realidad diversa de la violencia contra la mujer, lo que lleva a una confusión en el derecho internacional de los derechos humanos sobre los estándares conceptuales sobre la violencia de género, al alejarse del consenso doctrinal y de los criterios jurídicos que caracterizan a la violencia doméstica como una forma de violencia contra la mujer (Mercado Carmona, 2017: 237).

\section{Contenido de la Convención Belém do Pará}

Del contenido de la Convención Belém do Pará es en particular destacable la conceptualización de la violencia contra la mujer como una violencia estructural basada en el género; la vinculación relacional de la violencia contra la mujer y los derechos humanos; la inclusión de la doctrina de la «diligencia debida» por parte de los Estados y los mecanismos de control que contempla, conjugando órganos de control no convencionales y órganos de control convencionales. A continuación, veremos cómo se abordan en la Convención Belém do Pará.

\section{Violencia estructural basada en el género}

La violencia contra la mujer se plantea como una realidad sistémica estructural. En el preámbulo, al plantear los fundamentos de esta Convención, se señala que la violencia contra la mujer es «una manifestación de las relaciones de poder históricamente desiguales entre mujeres y hombres» (preámbulo, párrafo 3), para agregar luego, en su artículo 1, que «debe entenderse por violencia contra la mujer cualquier acción o conducta, basada en su género, que cause muerte, daño o sufrimiento, físico, sexual o sicológico a la mujer». Con estas disposiciones, determina la existencia de una violencia contra la mujer de carácter estructural, en tanto se funda en conceptos de inferioridad o subordinación de la mujer, configurados en el orden social (género), ${ }_{11}^{11}$ producto de las relaciones históricas de jerarquía y poder desigual que afectan a las mujeres.

11. La conducta social basada en el género expresa la relación social de jerarquía entre sexos, en que el sujeto es lo masculino y la alteridad lo femenino, lo que crea una relación jerarquizada de poder entre lo masculino y lo femenino (sujetos hombres y sujetos mujeres), que determina relaciones de dominio o de subordinación de carácter estructural que afecta a las mujeres (Iriarte Rivas, 2018: 37). 
Siguiendo a la teórica feminista Iris Marion Young, la conceptualización de esta violencia como estructural permite plantearla como una situación de injusticia igualmente estructural, en tanto se reproduce en forma sistemática en las principales instituciones políticas, económicas y culturales - sin que sea necesario identificar a un grupo con conciencia o intencionalidad de oprimir-, por el solo hecho de obtener un beneficio de la opresión del otro; la otra, en este caso. La dominación se expresa en las relaciones entre los grupos sociales en general, en las cuales el grupo oprimido es puesto en situaciones de injusticia o discriminación (Young, 2000: 71-103).

Por último, es interesante destacar que, al considerar la violencia contra la mujer como un fenómeno estructural, esta Convención plantea su abordaje como un todo que tiene diversas manifestaciones y expresiones, pero que no es parcelable como fenómeno. ${ }^{12}$ En este sentido, recepciona los estándares conceptuales sobre la violencia de género, lo que permite su comprensión fenomenológica en el abordaje jurídico, en particular en lo referente a la afectación de los derechos humanos de la mujer.

\section{Violencia contra la mujer y derechos humanos}

La concepción estructurante de la Convención de Belém do Pará determina que ésta contenga una vinculación relacional de la violencia contra la mujer y los derechos humanos. La vinculación de la violencia de género y los derechos humanos fue, como se dijo líneas arriba, un tema ausente del derecho internacional de los derechos humanos hasta la dictación de la Recomendación 19. Esta Convención continúa y afianza este planteamiento, al establecer en su articulado el capítulo 2, que se refiriere de modo expreso a los derechos protegidos por el texto convencional.

Para el análisis de estos artículos, los ordenaremos en dos grupos. Primero, el artículo 3 y artículo 6, que abren y cierran respectivamente este capítulo, se refieren al derecho de la mujer a una vida libre de violencia. El artículo 3 establece el derecho de la mujer a una vida libre de violencia tanto en el ámbito público como privado; el artículo 6 se refiere de un modo no taxativo al alcance de este derecho, que comprende el derecho a ser libre de toda forma de discriminación y ser valorada y educada libre de estereotipos y prácticas sociales basadas en conceptos de inferioridad o subordinación. Esta Convención recepciona así una de las propuestas planteadas por el ius feminismo en materia de derechos, que plantea la configuración de nuevos derechos humanos que permitan avanzar en la superación de modelos estructurales de discriminación y subordinación de las mujeres.

12. Como se señaló respecto de la violencia contra la mujer y la violencia doméstica, el Convenio de Estambul le otorga a esta última una categoría jurídica diferenciada de la violencia de género. Lo que, en cambio, no ocurre en la Recomendación 35, en que la violencia en razón de género contra la mujer es abordada como un fenómeno integral que se expresa en los diversos ámbitos sociales. 
En segundo lugar, los artículos 4 y 5 se refieren al reconocimiento y garantía de los derechos consagrados en el derecho internacional de los derechos humanos respecto de la mujer. El primer artículo consigna de modo expreso el derecho de toda mujer al reconocimiento, goce, ejercicio y protección de los derechos y libertades consagrados en los diversos instrumentos internacionales de derechos humanos; a continuación, de modo no taxativo, menciona derechos o grupos de derechos particularmente destacados en relación con la violencia de género y la afectación de derecho a las mujeres. El artículo 5, por su parte, expresa el reconocimiento a la mujer del ejercicio libre y pleno de sus derechos políticos, económicos, sociales y culturales; en ese contexto, plantea que los Estados partes reconocen que la violencia contra la mujer anula el ejercicio de estos derechos. Ambos artículos de la Convención Belém do Pará expresan, en el mismo sentido de la CEDAW y, en especial, de la Declaración y el Programa de Acción de Viena, ${ }^{13}$ el reconocimiento de que los derechos humanos de las mujeres y las niñas forman parte inalienable e indivisible de los derechos humanos universales (Iriarte Rivas, 2018: 102-103); y que, como se plantea en el preámbulo, la violencia de género es una ofensa a la dignidad humana y constituye una violación a los derechos y libertades de la mujer, por lo que para avanzar en la no discriminación y en respeto irrestricto de los derechos, los Estados partes deben progresar en la prevención, sanción y erradicación de la violencia contra la mujer.

\section{Inclusión de la doctrina de la «debida diligencia»}

La Convención Belém do Pará, siguiendo a la CEDAW y en particular a la Recomendación $19,{ }^{14}$ recepciona en su texto la doctrina de la «debida diligencia». Esto implicó en su momento romper con un importante paradigma de los derechos humanos referido al alcance de la responsabilidad de los Estados y sus obligaciones (Mejía Guerrero, 2012: 194-195). Estos ya no solo eran responsables de actos cometidos por sus propios agentes o representantes, sino también por actos cometidos por particulares, cuando no se hubieren tomado medidas con la diligencia debida orientadas a prevenir, sancionar y erradicar la violencia contra la mujer (artículo 7). La configuración de este estándar en esta Convención tiene dos elementos especialmente destacables. Por una parte, está la ampliación de la responsabilidad del Estado descrita en el pá-

13. El Programa de Acción de Viena fue aprobado por la Conferencia Mundial de Derechos Humanos el 25 de junio de 1993. Véase la Declaración y Programa de Acción de Viena en https://bit.ly/316VApI.

14. La CEDAW señala en su artículo 5 literal e, que los Estados partes se comprometen a tomar «las medidas apropiadas para eliminar la discriminación contra la mujer practicada por cualesquiera personas, organizaciones o empresas». La Recomendación 19, a su vez, en la Observación General 9, específica que, «en virtud del derecho internacional [...], los Estados también pueden ser responsables de actos privados si no adoptan medidas con la diligencia debida para impedir la violación de los derechos o para investigar y castigar los actos de violencia». 
rrafo anterior; pero, además, de ésta se deriva el segundo elemento a destacar, que supone dar cuenta de una realidad propia de la subjetividad del sujeto mujeres, que implica que los actos de violencia contra la mujer basada en el género son cometidos generalmente por particulares en la esfera privada del orden social, ${ }_{15}^{5}$ por lo que el Estado, en tanto garante y responsable de evitar la violación a los derechos y libertades de las personas, debe actuar en concordancia con esa realidad (diligencia debida), transformándose, de no ser así, en responsable de la ocurrencia de tales violaciones, las cometa un agente del Estado o un particular.

\section{Mecanismos de protección que contempla la Convención Belém do Pará}

Esta Convención contempla tres vías de protección, lo que convierte a este instrumento en uno de los más completos respecto del sistema de control. ${ }^{16}$ En primer lugar, está la elaboración de informes anuales nacionales que los Estados deben remitir a la Comisión Interamericana de Mujeres (CIM) (artículo 10). En segundo lugar, la denominada jurisdicción consultiva, que significa que los Estados partes y la CIM pueden requerir a la Corte Interamericana de Derechos Humanos (Corte IDH) una opinión consultiva sobre la interpretación de esta Convención (artículo 11). En tercer lugar, la posibilidad de la interposición de peticiones, por personas o grupos de personas o entidad no gubernamental, que contemplen denuncias o quejas a la Comisión Interamericana de Derechos Humanos (CIDH) por violación del artículo 7 de la Convención (artículo 12). Lo destacable de este último mecanismo es que permite a las mujeres víctimas de violencia por razones de género obtener amparo judicial internacional a través de las denuncias (Mercado Carmona, 2017: 229-230). En 2004, como reforzamiento de los mecanismos de seguimiento, se creó el Mecanismo de Seguimiento de la Convención Belém do Pará (MESECVI), que se constituye como un sistema consensuado e independiente para evaluar los avances de los Estados en el cumplimiento de la Convención (Mejía Guerrero, 2012: 209).

15. En ese sentido, el artículo 2 de la Convención complementa la conceptualización de violencia contenida en el artículo 1, al especificar que ésta también comprende la violencia contra la mujer basada en su género ocurrida dentro de la unidad doméstica o en la comunidad, o que sea perpetrada o tolerada por el Estado o sus agentes, donde quiera que ocurra. Es importante vincular esta última especificación (artículo 2 literal c) a lo referente a la responsabilidad del Estado, ya que, complementando lo establecido en el artículo 7 de la Convención, esta norma determina que se incluya como violencia la que sea tolerada por el Estado o sus agentes en cualquier lugar que ocurra.

16. La CEDAW contempla como mecanismo de seguimiento el Comité CEDAW, mientras que el Convenio de Estambul contempla un mecanismo de carácter no jurisdiccional, consistente en la presentación de informes por parte de los Estados partes ante un órgano de control creado para ello (Mercado Carmona, 2017: 231; Jiménez Sánchez, 2018: 497). 
La adopción de la Convención Belém do Pará, su contenido e implementación en relación con sus mecanismos de protección —en particular el del artículo 12, que permite presentar peticiones que contengan denuncias o quejas a la $\mathrm{CIDH}-$ ha permitido un importante desarrollo de un corpus de estándares de protección de los derechos humanos ante la violencia contra la mujer por razones de género. Analizar las decisiones del SIDH excede la posibilidad de este artículo; sin embargo, nos parece fundamental conocer, aunque sea en forma somera, las decisiones que, basándose en esta Convención, han aportado a la configuración de estándares del SIDH, como un modo de relevar los avances que la Convención Belém do Pará ha permitido en esta materia.

\section{María da Penha con Brasil ${ }^{17}$}

Este es el primer caso ante la CIDH que aborda la violencia contra la mujer (violencia doméstica) como una violación a los derechos humanos de las mujeres (fundamenta su argumentación en la Convención Belém do Pará). En lo fundamental, en esta resolución se establece el vínculo entra la discriminación y la violencia contra la mujer, agregando que la realidad de las víctimas es parte de un patrón (violencia estructural), respecto del cual el Estado no toma las medidas necesarias para enfrentarlo, lo que genera tolerancia del Estado e ineficacia judicial e institucional en los casos de violencia contra la mujer, en este caso violencia doméstica (Mejía Guerrero, 2012: 199).

\section{González y otras (Campo Algodonero) con México ${ }^{18}$}

Sin duda, este es el caso más paradigmático que hasta la fecha ha conocido y sentenciado la Corte IDH en materia de violencia contra la mujer fundada en el género. Es particularmente destacable porque examina el caso como situación estructural de violencia contra la mujer fundada en el género, determinando que el homicidio de las tres víctimas fue cometido por razones de género, por lo que constituyen casos de feminicidio; por lo mismo, la situación de las víctimas no fue analizada en virtud de las particularidades de este caso, sino que como integrantes de un colectivo más amplio que se ve afectado por una situación de violencia y desigualdad, lo que permite explicar la dimensión y ocurrencia de estos crímenes. Además, en este fallo se conceptualiza de un modo más preciso el estándar de la debida diligencia y el deber de protección estatal reforzado que estable la Convención, para lo cual determina los criterios jurídicos que permiten hacer responsable al Estado por crímenes cometidos

17. Caso 12.051, Maria da Penha Maia Fernandes. Informe 54/o1. Brasil, 16 de abril de 2001.

18. Sentencia del caso González y otras ("Campo Algodonero») con México, Corte Interamericana de Derechos Humanos, excepción preliminar, fondo, reparaciones y costas, 16 de noviembre de 2009. 
por particulares, recogiendo como fundamento la doctrina del riesgo previsible y evitable (Abramovich, 2010: 167-168).

\section{Penal Miguel Castro Castro con Perú19}

Es el primer caso que resolvió la Corte IDH en el cual aplicó la Convención Belém do Pará. En esta sentencia se establece la calificación jurídica de la violencia sexual cometida por agentes del Estado como tortura, fundada en la consideración de que los actos de agresión hacia las mujeres reclusas en el penal Castro Castro, en particular los de índole sexual, fueron de tal magnitud que atentaron contra su dignidad personal y que se utilizaron con el fin de castigar, intimidar, presionar, humillar y degradar a las mujeres, lo que permitió a la Corte señalar que allí se expresa un patrón de violencia contra la mujer en conflictos armados (Iriarte Rivas, 2017: 237).

\section{Casos Veliz Franco y otros con Guatemala ${ }^{20}$ y Velásquez Paiz con Guatemala ${ }^{21}$}

En ambas sentencias, la Corte determinó que en agresiones cometidas contra las mujeres se observa la manifestación de formas de violencia sexual o de actos de violencia de especial crueldad, brutalidad o ensañamiento, presentes en violencia por razones de género, lo cual permite determinar un criterio jurídico para calificar hechos de violencia contra mujeres como violencia por razones de género.

\section{Un paso más allá}

Para el cumplimiento de su objetivo de prevenir, sancionar y erradicar la violencia contra la mujer, la Convención refiere, en el capítulo 2, «Derechos protegidos», al derecho de la mujer a una vida libre de violencia (artículo 4), determinando luego -en una referencia no taxativa- que su alcance comprende el derecho de la mujer a ser libre de toda forma de discriminación y ser valorada y educada libre de estereotipos y prácticas sociales basadas en conceptos de inferioridad o subordinación (artículo 6). Del análisis del contenido de esta normativa, es posible establecer que la discriminación, los estereotipos y prácticas sociales basadas en el género constituyen violencia en contra de la mujer, según los términos en que está definida en el artículo 1, complementado por el artículo 2.

19. Sentencia del caso Penal Miguel Castro Castro con Perú, Corte Interamericana de Derechos Humanos, fondo, reparaciones y costas, 25 de noviembre de 2006.

20. Sentencia del caso Veliz Franco y otros con Guatemala, Corte Interamericana de Derechos Humanos, excepciones preliminares, fondo, reparaciones y costas, 19 de mayo de 2014, párr. 178.

21. Sentencia del caso Velásquez Paiz con Guatemala, Corte Interamericana de Derechos Humanos, excepciones preliminares, fondo, reparaciones y costas, 19 de noviembre de 2015, párr. 192. 
Respecto de la conceptualización de la discriminación referida en el artículo 6, un análisis sistemático del contenido de este instrumento internacional no puede sino determinar que se refiere a una concepción de discriminación estructural. En el preámbulo de la Convención, en su considerando tercero, se señala que la violencia contra la mujer es una manifestación de las relaciones de poder históricamente desiguales entre mujeres y hombres. Con ello está dando cuenta, como señaló, que el fundamento de la violencia está en las relaciones de jerarquía permanentes entre hombres y mujeres. Luego, en el artículo 1 de la Convención, al conceptualizar la violencia contra la mujer, se refiere a conductas basadas en su género, esto es, una configuración social, que a partir de la determinación cultural y social de lo masculino y lo femenino, dispone relaciones jerarquizada de sujetos hombres y sujetos mujeres, determinando la subordinación sistémica que afecta a las segundas. La Recomendación 35, en su considerando décimo, lo plantea de la siguiente manera: «La violencia por razón de género contra la mujer es uno de los medios sociales, políticos y económicos fundamentales a través de los cuales se perpetua la posición subordinada de la mujer con respecto al hombre». ${ }^{22}$

Asimismo, en su preámbulo, considerando segundo, la Convención señala que la violencia contra la mujer constituye la violación de los derechos humanos de éstas y la limitación total o parcial del reconocimiento (titularidad), goce y ejercicio de estos derechos y las libertades fundamentales, idea que se ve reforzada en el considerando siguiente, en el que se consigna que la violencia es una ofensa a la dignidad humana. En este planteamiento se encuentra el fundamento de que la violencia contra la mujer constituye discriminación, ya que, como señala la CEDAW en su artículo 1, la expresión «discriminación contra la mujer» denotará aquello que tenga por objeto o por resultado menoscabar o anular el reconocimiento, goce o ejercicio por la mujer de los derechos humanos y las libertades fundamentales.

La discriminación producto de la violencia contra la mujer expresará los elementos sistémicos (estructurales) propios de la violencia por razón de género, ya que esta discriminación no es producto de actos individuales, sino que la manifestación de realidades sociales determinadas por el género. ${ }^{23}$ Dicho de otro modo, la discriminación fundada en el género es producto de la subordinación sistémica de las mujeres (Iriarte Rivas, 2017: 134-135).

En el capítulo de derechos protegidos, la Convención, junto con referir al derecho

22. El considerando décimo noveno de la Recomendación 35 refuerza el planteamiento transcrito cuando señala «que la violencia por razón de género contra la mujer está arraigada en factores relacionados con el género, como la ideología del derecho y el privilegio de los hombres respecto de las mujeres, las normas sociales relativas a la masculinidad y la necesidad de afirmar el control o poder masculinos». Véase Recomendación General 35.

23. Recomendación General 35, considerando noveno. 
a una vida libre de violencia, plantea que la mujer tiene derecho al reconocimiento, goce, ejercicio y protección de los derechos y libertades consagrados en los diversos instrumentos internacionales de derechos humanos (artículo 4). Además, plantea que la mujer podrá ejercer libre y de modo pleno sus derechos políticos, económicos, sociales y culturales, establecidos en dichos instrumentos internacionales, agregando que los Estados partes reconocen que la violencia contra la mujer anula el ejercicio de estos derechos (artículo 5). Ambos artículos - en concordancia al ya señalado reconocimiento contenido en el preámbulo, de que la violencia contra la mujer constituye una violación a los derechos humanos que limita total y parcialmente el reconocimiento, goce y ejercicio de estos derechos y que constituye una ofensa a la dignidad- no hacen más que reforzar el reconocimiento contenido en diversos instrumentos internacionales de que los derechos humanos de las mujeres y las niñas forman parte inalienable e indivisible de los derechos humanos universales. ${ }^{24}$ En este sentido, la Recomendación 35, en el numeral decimoquinto, señala que «el derecho de las mujeres a una vida libre de violencia por razón de género es indivisible e independiente de otros derechos humanos».

El capítulo de derechos protegidos se inicia con la referencia al derecho de la mujer a un mundo libre de violencia. Luego establece la vinculación específica de la violencia contra la mujer con la afectación de los derechos consagrados en los instrumentos internacionales. Cierra con el artículo 6, que especifica el contenido del derecho de toda mujer a una vida libre de violencia. Esta lógica de ordenación expresa uno de los planteamientos estructurantes de la Convención: que el reconocimiento a una vida libre de violencia - basada en su género- significa, por una parte, el derecho al reconocimiento, goce ejercicio y protección de los derechos del Sistema Internacional de los Derechos Humanos - los derechos humanos de la mujer y las niñas forman parte inalienable e indivisibles de los derechos humanos universales-, pero que también significa el derecho a ser libre de las normas, conductas sociales y estereotipos que permiten el privilegio masculino respecto de las mujeres, que afirman el control o poder masculinos o que perpetúan la posición subordinada de la mujer con respecto al hombre.

Conforme con lo analizado, la discriminación estructural contra la mujer es constitutiva de violencia contra la mujer por razones de género en los términos que contempla la propia Convención. Por lo mismo, la materialidad de la discriminación estructural, como son los medios sociales, políticos y económicos a través de los cuales se perpetúa la posición subordinada de la mujer, deben ser prohibidos y erradicados en los términos que plantean la Convención Belém do Pará y los instrumentos del derecho internacional de los derechos humanos que la complementan.

24. El primer instrumento internacional en establecer este reconocimiento fue la Declaración y Programa de Acción de Viena de la Conferencia Mundial de Derechos Humanos, en junio de 1993. 


\section{A modo de conclusión}

La Convención de Belém do Pará fue el primer instrumento internacional de carácter convencional que abordó los derechos humanos de las mujeres y la violencia de género. En particular, vincula esta temática con la afectación que la violencia implica al reconocimiento, goce y ejercicio de los derechos humanos y libertades fundamentales; conceptualiza la violencia contra la mujer fundada en el género como violencia estructural; recepciona la doctrina de la «diligencia debida» por parte de los Estados; establece mecanismos de control que conjugan órganos de control no convencionales y órganos de control convencionales e, incluso, plantea nuevas perspectivas en materia de derechos humanos de la mujer al reconocer el derecho de toda mujer a una vida libre de violencia y establecer la prohibición expresa al ejercicio de violencia basada en el género.

El reconocimiento y alcance del planteamiento de que la mujer tiene derecho a una vida libre de violencia en la Convención Belém do Pará significa, por una parte, el reconocimiento de que los derechos humanos de la mujer y las niñas forman parte inalienable e indivisibles de los derechos humanos universales. Además, consagra el derecho a ser libre de las normas, conductas sociales y estereotipos que perpetúan la posición subordinada de la mujer con respecto al hombre. Esto último permite establecer que la discriminación estructural contra la mujer es constitutiva de violencia contra la mujer por razones de género en los términos que contempla la propia Convención, pudiendo y debiendo los Estados partes afrontar la materialidad de la discriminación estructural utilizando en toda su extensión a la Convención Belém do Pará.

\section{Referencias}

Abramovich, Víctor (2010). «Responsabilidad estatal por violencia de género: Comentarios sobre el caso "Campo Algodonero" en la Corte Interamericana de Derechos Humanos». Anuario de Derechos Humanos, 6: 167-182. DOI: 10.5354/0718-2279.2011.11491.

IRIARTE RIVAS, Claudia (2017). «La ausencia del sujeto mujeres en la configuración del sujeto jurídico: Buscando caminos hacia la igualdad sustancial de mujeres y hombres». Tesis para optar al grado de doctora en Derecho, Universidad de Chile. Disponible en https://bit.ly/2 $\mathrm{YF}_{5} \mathrm{VzV}$.

Jiménez SÁnchez, Carolina (2018). «Los derechos humanos de las mujeres en Europa y América Latina: Perspectiva jurisprudencial internacional». Araucaria, 20 (40): 483-510. DOI: 10.12795/araucaria.2018.i40.22.

Mejía Guerrero, Luz Patricia (2012). «La Comisión Interamericana de Mujeres y la Convención Belém do Pará: Impacto en el Sistema Interamericano de Derechos Humanos». Revista IIDH, 56: 189-213. Disponible en https://bit.ly/3j77Jtd. 
Mercado Carmona, Carmen (2017). «La erradicación de la violencia contra la mujer "por tratado": Un análisis comparado del Convenio de Estambul y de la Convención de Belén do Pará». Revista Europea de Derechos Fundamentales, 30: 213-339. Disponible en https://bit.ly/3liw6dz.

Ventura Franch, Asunción (2016). «El Convenio de Estambul y los sujetos de la violencia de género: El cuestionamiento de la violencia doméstica como categoría Jurídica». UNED: Revista de Derecho Público, 97: 179-208. DOI: 10.5944/ rdp.97.2016.17622.

Young, Iris Marion (2000). La justicia y la política de la diferencia. Valencia: Cátedra, Universitat de Vàlencia, Instituto de la Mujer.

\section{Sobre la autora}

Claudia Iriarte Rivas es abogada. Doctora en Derecho, investigadora en el Centro de Derechos Humanos de la Facultad de Derecho Universidad de Chile. Su correo electrónico es ciriarter@derecho.uchile.cl. (D) https://orcid.org/00oo-0003-0449-926X. 
El Anuario de Derechos Humanos es una publicación semestral de referencia y consulta en materia de derechos humanos y campos afines. Busca ser un espacio de discusión de los temas centrales en el ámbito nacional e internacional sobre derechos humanos. Es publicado desde 2005 por el Centro de Derechos Humanos de la Facultad de Derecho de la Universidad de Chile.

\author{
EDITORA GENERAL \\ Claudia Iriarte Rivas \\ ciriarter@derecho.uchile.cl \\ EDITORA DE ESTE NÚMERO \\ Liliana Galdámez Zelada \\ lgaldamez@derecho.uchile.cl \\ SITIO WEB \\ anuariocdh.uchile.cl \\ CORREO ELECTRÓNICO \\ anuario-cdh@derecho.uchile.cl \\ LICENCIA DE ESTE ARTÍCULO
}

Creative Commons Atribución Compartir Igual 4.o Internacional

\author{
कै \\ La edición de textos, el diseño editorial \\ y la conversión a formatos electrónicos de este artículo \\ estuvieron a cargo de Tipográfica \\ (www.tipografica.io)
}

